\title{
AUTOAVALIAÇÃO NEGATIVA DA SAÚDE EM TRABALHADORAS DE ENFERMAGEM DA ATENÇÃO BÁSICA
}

\author{
POOR SELF-ASSESSMENT OF THE HEALTH OF PRIMARY \\ HEALTH CARE NURSING WORKERS
}

\author{
AUTOEVALUACIÓN NEGATIVA DE LA SALUD EN TRABAJADORAS \\ DE ENFERMERÍA DE LA ATENCIÓN BÁSICA
}

Iracema Lua ${ }^{1}$

Maura Maria Guimarães de Almeida²

Tânia Maria de Araújo ${ }^{3}$

Jorgana Fernanda de Souza Soares ${ }^{4}$

Kionna Oliveira Bernardes Santos ${ }^{5}$

Resumo O objetivo deste estudo é avaliar os fatores associados à autoavaliação negativa da saúde entre trabalhadoras de enfermagem. Realizou-se estudo transversal exploratório, com amostra probabilística de 451 trabalhadoras de enfermagem da Atenção Básica à Saúde. Foi aplicado questionário com informações sociodemográficas, de hábitos de vida, ocupacionais, aspectos psicossociais e satisfação no trabalho e morbidade das trabalhadoras. A autoavaliação da saúde foi aferida por meio da questão "De modo geral, em comparação às pessoas da sua idade, como você considera o seu próprio estado de saúde?". Os dados foram analisados por meio do modelo de regressão logística em blocos. Identificou-se prevalência de 15,8\% de autoavaliação negativa da saúde. Os fatores associados foram: incompatibilidade das atividades desenvolvidas com o cargo, média e alta sobrecarga doméstica, situação de trabalho ativo (alto controle, alta demanda), avaliação ruim da qualidade de vida e transtornos mentais comuns. Os achados corroboram a relação entre processo saúdedoença e condições de vida e trabalho e indicam a necessidade de políticas públicas de prevenção e promoção da saúde das trabalhadoras de enfermagem. Essas politicas, ao constituir situações favoráveis de trabalho, podem ser muito relevantes para a qualidade da assistência prestada aos usuários do Sistema Único de Saúde.

Palavras-chave autoavaliação; enfermagem; atenção básica à saúde; saúde do trabalhador; trabalho feminino.
Abstract The goal of this study is to assess the elements associated with the negative self-assessment of health among nursing workers. We performed a cross-sectional exploratory study, with a probability sample composed of 451 Primary Health Care nursing workers. We applied a questionnaire with sociodemographic data, as well as data on life habits, occupations, psychosocial aspects and satisfaction at work and morbidity of the workers. The self-assessment of health was measured through the question "Overall, in comparison with other people your age, how would you consider your own health status?" The data was analyzed using the model of logistic regression in blocks. We identified a prevalence of $15.8 \%$ of negative health self-assessments. The factors associated to it were: incompatibility of the activities developed with the position, medium and high housework overload, active work situation (high control, high demand), poor assessment of the quality of life, and common mental disorders. The findings confirm the relationship between the health-disease process and the life and working conditions, and point to a need for public policies of prevention and promotion of the health of nursing workers. By constituting favorable work situations, these policies may be highly relevant for the quality of the care provided to the users of the Brazilian Unified Health System (Sistema Único de Saúde, SUS, in the Portuguese acronym).

Keywords self-assessment; nursing; primary health care; health of the worker; female labor. 


\section{Introdução}

A Atenção Básica é a porta de entrada do sistema de saúde brasileiro; tem por finalidade descentralizar os serviços, organizar o modelo assistencial, reafirmar e incorporar as diretrizes e princípios básicos do Sistema Único de Saúde (SUS): acessibilidade, vínculo, continuidade e longitudinalidade do cuidado, com atenção centrada na pessoa, não na doença (Brasil, 2012).

O trabalho na Atenção Básica é desenvolvido por equipes multiprofissionais, dentre os quais se encontram enfermeiros(as), técnicos(as) e auxiliares de enfermagem que desenvolvem atividades administrativas e de supervisão do trabalho, além da assistência de enfermagem, e ações de prevenção de doenças, promoção e vigilância à saúde, atividades educativas e políticas, dentre outras (Brasil, 2012). Devido à execução dessas atividades, esses trabalhadores são expostos a fatores físicos, químicos, biológicos, mecânicos, psicossociais e ergonômicos que podem afetar a sua saúde e, por conseguinte, a sua autoavaliação sobre ela.

O presente estudo está baseado no conceito ampliado de saúde, como o "estado de completo bem-estar biopsicossocial" (Organização Mundial da Saúde - OMS). Portanto, mensurá-la é uma tarefa difícil, devido a sua heterogeneidade e à amplitude de conceitos envolvidos em sua definição, o que torna sua avaliação/mensuração complexa. A autoavaliação é referenciada pela OMS como um indicador confiável, válido e relevante para identificar a situação de saúde de indivíduos e populações (Silva, 2012).

Este indicador apresenta uma classificação global do indivíduo que considera sinais e sintomas de doenças, além do impacto dessas condições sobre seu bem-estar físico, mental e social (Pagotto, Nakatani e Silveira, 2011). Pode ser realizada de maneira simples, baseada em uma única questão. A adoção desse indicador simples vem sendo amplamente empregada para a autoavaliação da saúde (Silva, 2012).

Diversos autores, nacionais e internacionais, empregaram esta medida em estudos de população geral (Peres et al., 2010; Traebert, Bortoluzzi e Kehrig, 2011; Pavão, Werneck e Campos, 2013; Wu et al., 2013; Ergin e Mandiracioglu, 2015) ou de grupos ocupacionais específicos (Pappas, Alamanos e Dimoliatis, 2005; Costa, 2010; Fernandes et al., 2010; Garcia, Höfelmann e Facchini, 2010; Griep et al., 2011; Malinauskiene et al., 2011; Guimarães et al., 2012; Silva, 2012; Silva e Barreto, 2012; Porto et al., 2013; Theme-Filha, Costa e Guilam, 2013; Hämmig e Bauer, 2014; Rocha et al., 2014; Bryła, Szymocha e Maniecka-Bryła, 2015; Petarli et al., 2015). Os resultados desses estudos apontaram associação dessa medida com as condições de vida, características sociodemográficas e comportamentais, de saúde e do ambiente e organização do trabalho. 
Entretanto, ainda há poucos estudos (Fernandes et al., 2010; Garcia, Höfelmann e Facchini, 2010; Porto et al., 2013) que abordam a autoavaliação do estado de saúde em condições específicas do trabalho em enfermagem na Atenção Básica, assim como os seus respectivos fatores associados.

Reconhecendo a importância fundamental do cuidado prestado pelas equipes de enfermagem na rede primária de saúde para a eficácia das políticas públicas e implementação do SUS, este estudo objetivou avaliar os fatores associados à autoavaliação negativa da saúde entre trabalhadoras de enfermagem na Atenção Básica.

A identificação das características da população trabalhadora que estão associadas a um estado negativo de saúde pode contribuir para gerar novas hipóteses a serem testadas em estudos longitudinais, base importante para ações de promoção da saúde nessa população.

\section{Percurso metodológico}

Este estudo é um recorte do projeto multicêntrico "Condições de trabalho, condições de emprego e saúde dos trabalhadores da saúde na Bahia", desenvolvido pelo Núcleo de Epidemiologia da Universidade Estadual de Feira de Santana em parceria com a Universidade Estadual do Sudoeste da Bahia, Universidade Estadual de Santa Cruz, Universidade Federal da Bahia e Universidade Federal do Recôncavo Baiano.

Realizou-se estudo epidemiológico de corte transversal, exploratório, com trabalhadoras de enfermagem da Atenção Básica de cinco municípios baianos, a saber: Feira de Santana, Distrito Sanitário do Centro Histórico em Salvador, Jequié, Santo Antônio de Jesus e Itabuna. Por considerar que o trabalho e a saúde são categorias de estudo que estão intrinsecamente afetadas pelas condições de gênero, optou-se por estudar separadamente os trabalhadores e as trabalhadoras de enfermagem, e delimitou-se, para este estudo, a análise da população feminina.

A amostra probabilística foi calculada com base na população total de trabalhadores de enfermagem $(\mathrm{N}=938)$, usando a prevalência esperada de autoavaliação negativa de saúde de 21,86\% (Garcia, Höfelmann e Facchini, 2010), precisão de 3\% e intervalo de $95 \%$ de confiança, estimou-se o tamanho amostral em 411 participantes. Acrescentou-se 20\% (n=82) para compensar possíveis perdas e recusas, o que resultou em 493 trabalhadoras selecionadas por meio de lista de números aleatórios. Excluíram-se aquelas que estavam afastadas do trabalho por licença ou férias. No caso de insucesso após três tentativas de entrevista houve substituição por outra profissional com características similares (categoria profissional, idade e local de trabalho). Investigou-se um total de 451 trabalhadoras de enfermagem da Atenção Básica. 


\section{Variáveis de estudo}

Para a coleta de dados empregou-se um questionário construído com base na revisão de literatura, previamente testado em estudo piloto. O questionário incluiu características sociodemográficas, hábitos de vida, características do trabalho, aspectos psicossociais do trabalho, satisfação no trabalho e situação de saúde, agrupadas em blocos de análise. Algumas dessas variáveis são indicadores compostos. Por exemplo, a "sobrecarga doméstica", é o somatório das tarefas domésticas básicas (lavar, passar, limpar, cozinhar), ponderado pelo número de moradores, aplicada a fórmula: $\mathrm{SD}=$ (lavar + passar $+\mathrm{lim}$ par + cozinhar) x (M-1) (Aquino, 1996), categorizada em tercis: alta, média e baixa sobrecarga, conforme sugerido por Pinho e Araújo (2012).

Outra variável é constituída pelo Modelo Demanda-Controle de Karasek, que permite a construção de quatro categorias de trabalho, baseado na combinação das dimensões demanda psicológica e controle sobre o trabalho, dicotomizadas pela média em alto e baixo: baixa exigência (baixo controle e baixa demanda), trabalho ativo (baixo controle e alta demanda), trabalho passivo (alto controle e baixa demanda) e alta exigência (alto controle e alta demanda). A principal predição estabelecida pelo modelo é que a situação de alta exigência no trabalho é fator de risco para os agravos ocupacionais decorrentes dos fatores psicossociais, tais como fadiga, ansiedade, depressão e doença física (Araújo et al., 2003).

Já os Transtornos Mentais Comuns (TMC) é uma variável avaliada pelo Self-Reporting Questionnaire-SRQ-20, instrumento validado nacionalmente e com desempenho satisfatório, sendo sete (7) ou mais respostas positivas o melhor ponto de corte para as mulheres (Santos et al., 2010).

A variável desfecho foi a autoavaliação negativa do estado de saúde, obtida por meio da questão "De modo geral, em comparação às pessoas da sua idade, como você considera o seu próprio estado de saúde?" As respostas foram dicotomizadas em: positiva (muito bom/bom) e negativa (regular/ ruim/muito ruim).

\section{Análise dos dados}

Inicialmente foi feita análise descritiva, seguida de análise bivariada, com adoção de critério de pré-seleção, para a análise multivariada, do valor de $p \leq 0,25$, estimado pelo teste qui-quadrado de Pearson ou teste exato de Fisher. Levou-se em consideração ainda a consistência das associações encontradas na literatura. Na análise multivariada, empregou-se a regressão logística, processada em etapas: Pré-seleção das variáveis em cada bloco de dados separadamente, com critério de retenção para as associações com valores de $\mathrm{p} \leq 0,17$; Seleção de variáveis interblocos - análise multivariada 
entre os blocos, de acordo com a seguinte ordem de entrada das variáveis: sociodemográficas, hábitos de vida, trabalho (características ocupacionais e aspectos psicossociais), satisfação no trabalho e situação de saúde; Obtenção do modelo final - no qual permaneceram no modelo final as variáveis que foram associadas estatisticamente à autoavaliação negativa do estado de saúde ao nível de significância de $5 \%$ (valor de $\mathrm{p} \leq 0,05$ ).

A medida de associação empregada foi a razão de prevalência (RP), obtida por meio da Regressão de Poisson com variância robusta, assim como seus respectivos intervalos de confiança (Coutinho, Scazufca e Menezes, 2008; Francisco et al., 2008).

O teste de bondade de ajuste do modelo (teste de Hosmer e Lemeshow goodness of fit) e a área sob a curva ROC foram utilizados para a avaliação da adequação do modelo final de regressão e, em seguida, avaliados os modelos com análise dos padrões de observação influentes.

O projeto multicêntrico do qual faz parte este estudo foi previamente aprovado pelo Comitê de Ética em Pesquisa da Universidade Estadual de Feira de Santana, de acordo com o protocolo n. 081/2009, CAAE 0086.0.059.000-09. A coleta de dados foi padronizada com base no "Manual de procedimentos e técnicas", com treinamento dos pesquisadores quanto aos aspectos éticos da pesquisa com seres humanos (resolução n. 466/12 do CNS). Os profissionais selecionados foram abordados no seu ambiente de trabalho, informados a respeito da pesquisa, sendo solicitada a assinatura do termo de consentimento livre e esclarecido.

\section{Resultados da análise descritiva}

Foram estudadas 451 trabalhadoras de enfermagem da Atenção Básica da Bahia, com características predominantes de idade superior a 40 anos $(63,1 \%)$, cor da pele não branca $(79,1 \%)$, com companheiro $(52,6 \%)$ e filhos $(60,9 \%)$. Quanto aos hábitos de vida, 83,8\% realizavam atividades regulares de lazer, 61,6\% não realizavam atividade física, 59,4\% não consumiam bebidas alcoólicas e 96,6\% não eram fumantes.

Os dados sobre as características do trabalho demonstraram que as trabalhadoras de enfermagem da Atenção Básica tinham menos de cinco anos de trabalho $(67,4 \%)$, um único vínculo $(63,8 \%)$, trabalho precarizado (prestador de serviços, cargo de confiança, cooperativado e terceirizado, 52,9\%), com direitos trabalhistas parciais $(71,2 \%)$, eram as principais responsáveis pelas atividades domésticas (54,5\%) e estavam expostas à média ou alta sobrecarga doméstica $(68,9 \%)$.

No que tange aos aspectos psicossociais do trabalhado, 43,9\% das trabalhadoras de enfermagem estavam expostas a situações de trabalho que traziam risco para o adoecimento, segundo o modelo demanda-controle de Karasek: 20,3\% 
estavam expostas ao trabalho de alta exigência, situação de maior exposição, e 23,6\% expostas ao trabalho passivo. Quanto ao apoio social, 46,8\% tinham um baixo apoio dos colegas e supervisores; $46,3 \%$ se comprometiam excessivamente com o seu trabalho; $49,5 \%$ estavam expostas ao alto esforço envolvido na realização do trabalho e $43,8 \%$ à baixa recompensa propiciada pelo trabalho.

\section{Análise bivariada}

A prevalência de autoavaliação negativa do estado de saúde foi de $15,8 \%$, com maior frequência entre as trabalhadoras com mais de 40 anos de idade $(20,1 \%)$, de raça/cor da pele branca $(19,4 \%)$, com companheiros $(17 \%)$, com filhos $(18,8 \%)$, que não praticavam atividades de lazer $(19,7 \%)$, não realizavam atividade física $(16,7 \%)$, não consumiam bebidas alcoólicas $(17,6 \%)$ e eram fumantes $(21,4 \%)$ (Tabela 1$)$. Com base nos critérios preestabelecidos, foram selecionadas, para a análise multivariada, as variáveis idade, filhos, atividade física e uso de bebidas alcoólicas, acrescidas, ainda, das variáveis com evidências de associação estatística em outros estudos, como situação conjugal (Pappas, Alamanos e Dimoliatis, 2005; Costa, 2010; Pagotto, Nakatani e Silveira, 2011; Pavão, Werneck e Campos, 2013; Rocha et al., 2014; Ergin e Mandiracioglu, 2015) e raça/cor da pele (Costa, 2010; Peres et al., 2010; Pagotto, Nakatani e Silveira, 2011; Pavão, Werneck e Campos, 2013).

\section{Tabela 1}

Autoavaliação da saúde segundo características sociodemográficas e hábitos de vida em trabalhadoras de enfermagem da atenção básica. Bahia, Brasil, 2011-2012

\begin{tabular}{|c|c|c|}
\hline \multirow[b]{2}{*}{ Variáveis (n) } & \multicolumn{2}{|c|}{ Autoavaliação do estado de saúde } \\
\hline & $\begin{array}{l}\text { Positiva } \\
\text { n (\%) }\end{array}$ & $\begin{array}{l}\text { Negativa } \\
\mathrm{n}(\%)\end{array}$ \\
\hline \multicolumn{3}{|l|}{ Sociodemográficas } \\
\hline \multicolumn{3}{|l|}{ Idade $(444)^{*}$} \\
\hline Até 40 anos & $243(86,8)$ & $37(13,2)$ \\
\hline Mais de 40 anos & $131(79,9)$ & $33(20,1)$ \\
\hline \multicolumn{3}{|l|}{ Cor da pele (445) } \\
\hline Branca & $75(80,6)$ & $18(19,4)$ \\
\hline Não-branca & $299(84,9)$ & $53(15,1)$ \\
\hline \multicolumn{3}{|l|}{ Situação conjugal (449) } \\
\hline Com companheiro & $196(83)$ & $40(17)$ \\
\hline Sem companheiro & $182(85,4)$ & $31(14,6)$ \\
\hline \multicolumn{3}{|l|}{ Filhos $(448)^{*}$} \\
\hline Não & $155(88,6)$ & $20(11,4)$ \\
\hline Sim & $222(81,2)$ & $51(18,8)$ \\
\hline \multicolumn{3}{|l|}{ Hábitos de vida } \\
\hline \multicolumn{3}{|c|}{ Prática de atividades de lazer (445) } \\
\hline Sim & $316(84,7)$ & $57(15,3)$ \\
\hline Não & $58(80,3)$ & $14(19,7)$ \\
\hline \multicolumn{3}{|l|}{ Prática de atividade física $(401)^{*}$} \\
\hline Sim & $136(88,3)$ & $18(11,7)$ \\
\hline Não & $206(83,3)$ & $41(16,7)$ \\
\hline \multicolumn{3}{|l|}{ Uso de bebidas alcoólicas (394)* } \\
\hline Não & $193(82,4)$ & $41(17,6)$ \\
\hline Sim & $141(88,1)$ & $19(11,9)$ \\
\hline \multicolumn{3}{|l|}{ Tabagismo (443) } \\
\hline Não fumante & $361(84,3)$ & $67(15,7)$ \\
\hline Fumante atual & $12(78,6)$ & $3(21,4)$ \\
\hline
\end{tabular}


No que se refere ao trabalho, maiores frequências de autoavaliação negativa da saúde foram observadas entre as técnicas/auxiliares de enfermagem $(18,3 \%)$, com mais de dez anos de trabalho $(23,8 \%)$ e mais de dez anos de trabalho na unidade $(19,6 \%)$, concursada/celetista $(17,2 \%)$, com outro vínculo de trabalho (16\%), direitos trabalhistas parciais $(16,9 \%)$, jornada de trabalho maior que 60 horas semanais $(21,6 \%)$, alta demanda física no trabalho $(19,4 \%)$, com atividades incompatíveis com o cargo $(36,4 \%)$, com segurança pessoal ameaçada no trabalho $(19,3 \%)$, que realizavam trabalho doméstico $(20,2 \%)$ e tinham alta sobrecarga doméstica $(21,4 \%)$ (Tabela 2$)$. De acordo com os critérios de seleção adotados, foram selecionadas, para a análise multivariada, as variáveis categoria profissional, tempo de trabalho, jornada de trabalho semanal, demanda física sobre o trabalho, compatibilidade das atividades desenvolvidas, segurança pessoal ameaçada no trabalho, trabalho doméstico e sobrecarga doméstica.

Tabela 2

Autoavaliação da saúde segundo características do trabalho de enfermagem da atenção básica. Bahia, Brasil, 2011-2012

\begin{tabular}{|c|c|c|}
\hline \multirow[b]{2}{*}{ Variáveis da característica do trabalho (n) } & \multicolumn{2}{|c|}{ Autoavaliação do estado de saúde } \\
\hline & $\begin{array}{l}\text { Positiva } \\
\text { n (\%) }\end{array}$ & $\begin{array}{l}\text { Negativa } \\
\text { n (\%) }\end{array}$ \\
\hline \multicolumn{3}{|l|}{ Categoria profissional (451)* } \\
\hline $\begin{array}{l}\text { Enfermeira } \\
\text { Técnica/auxiliar de enfermagem }\end{array}$ & $\begin{array}{l}134(89,3) \\
246(81,7)\end{array}$ & $\begin{array}{l}16(10,7) \\
55(18,3)\end{array}$ \\
\hline \multicolumn{3}{|l|}{ Tempo de trabalho $(448)^{*}$} \\
\hline Menos de 5 anos & $152(88,9)$ & $19(11,1)$ \\
\hline De 5 a 10 anos & $97(88,9)$ & $12(11,1)$ \\
\hline Mais de 10 anos* $^{*}$ & $128(76,2)$ & $40(23,8)$ \\
\hline \multicolumn{3}{|l|}{ Tempo de trabalho na unidade (448) } \\
\hline Menos de 5 anos & $255(84,4)$ & $47(15,6)$ \\
\hline De 5 a 10 anos & $81(85,1)$ & $14(14,9)$ \\
\hline Mais de 10 anos & $41(80,4)$ & $10(19,6)$ \\
\hline \multicolumn{3}{|l|}{ Vínculo de trabalho (446) } \\
\hline \multirow{2}{*}{$\begin{array}{l}\text { Concursada/ celetista } \\
\text { Trabalho precarizado (prestador de serviços/cargo de } \\
\text { confiança/ cooperativado/terceirizado) }\end{array}$} & $174(82,8)$ & $36(17,2)$ \\
\hline & $202(85,6)$ & $34(14,4)$ \\
\hline \multicolumn{3}{|l|}{ Possui outro vínculo (450) } \\
\hline Não & $242(84,3)$ & $45(15,7)$ \\
\hline Sim & $137(84)$ & $26(16)$ \\
\hline \multicolumn{3}{|l|}{ Possui direitos trabalhistas (441) } \\
\hline Sim, todos & $86(86)$ & $14(14)$ \\
\hline Sim, parcialmente & $261(83,1)$ & $53(16,9)$ \\
\hline Não, nenhum & $24(92,3)$ & $2(7,7)$ \\
\hline \multicolumn{3}{|l|}{ Jornada de trabalho semanal (435)* } \\
\hline Até 40 horas & $261(85,6)$ & $44(14,4)$ \\
\hline Entre 41 e 60 horas & $65(82,3)$ & $14(17,7)$ \\
\hline Mais de 60 horas & $40(78,4)$ & $11(21,6)$ \\
\hline \multicolumn{3}{|l|}{ Demanda física sobre o trabalho $(447)^{*}$} \\
\hline Baixa demanda física & $218(86,9)$ & $33(13,1)$ \\
\hline \multirow{2}{*}{\multicolumn{3}{|c|}{ Compatibilidade das atividades desenvolvidas $(449)^{*}$}} \\
\hline & & \\
\hline Compatíveis com o cargo & $364(85,2)$ & $63(14,8)$ \\
\hline Não compatíveis com o cargo & $14(63,6)$ & $8(36,4)$ \\
\hline \multicolumn{3}{|l|}{ Segurança pessoal ameaçada no trabalho $(450)^{*}$} \\
\hline Não & $241(86,4)$ & $38(13,6)$ \\
\hline Sim & $138(80,7)$ & $33(19,3)$ \\
\hline \multicolumn{3}{|l|}{ Trabalho doméstico (448)* } \\
\hline Não & $183(89,7)$ & $21(10,3)$ \\
\hline Sim & $195(79,8)$ & $49(20,2)$ \\
\hline \multicolumn{3}{|l|}{ Sobrecarga doméstica $(440)^{*}$} \\
\hline Baixa sobrecarga & $127(92,7)$ & $10(7,3)$ \\
\hline Média sobrecarga & $112(82,8)$ & $23(17,2)$ \\
\hline Alta sobrecarga & $132(78,6)$ & $36(21,4)$ \\
\hline
\end{tabular}

Fonte: Os autores.

* Valor de $\mathrm{p} \leq 0,25$. 
No que tange aos aspectos psicossociais do trabalho, observaram-se maiores frequências de autoavaliação negativa entre trabalhadoras com baixo apoio social $(16,7 \%)$, comprometimento excessivo com o trabalho $(19,1 \%)$, situação de trabalho ativo $(22,8 \%)$, alto esforço na realização do trabalho $(21,4 \%)$ e alta recompensa propiciada pelo trabalho $(16,7 \%)$ (Tabela 3). Selecionaram-se para a análise multivariada, segundo os critérios adotados, as variáveis modelo demanda-controle, esforço envolvido na realização do trabalho e comprometimento excessivo com o trabalho. Apesar dos estratos de baixo apoio social e alta recompensa propiciada pelo trabalho terem apresentado maiores prevalência de autoavaliação negativa do que aqueles que tinham alto apoio e baixa recompensa, as diferenças não foram estatisticamente significantes. Portanto, não foram observadas diferenças expressivas entre os estratos dessa variável; ou seja, essas diferenças podem decorrer de variações do acaso. Desse modo, não atendiam aos critérios de retenção de variáveis previamente definidos.

Tabela 3

Autoavaliação da saúde segundo aspectos psicossociais do trabalho em trabalhadoras de enfermagem da atenção básica. Bahia, Brasil, 2011-2012

\begin{tabular}{|c|c|c|}
\hline \multirow[b]{2}{*}{ Variáveis psicossociais do trabalho (n) } & \multicolumn{2}{|c|}{ Autoavaliação do estado de saúde } \\
\hline & $\begin{array}{l}\text { Positiva } \\
\text { n (\%) }\end{array}$ & $\begin{array}{l}\text { Negativa } \\
\text { n (\%) }\end{array}$ \\
\hline \multicolumn{3}{|l|}{ Apoio social (423) } \\
\hline Alto apoio & $191(84,8)$ & $34(15,2)$ \\
\hline Baixo apoio & $165(83,3)$ & $33(16,7)$ \\
\hline \multicolumn{3}{|c|}{ Comprometimento excessivo com o trabalho $(441)^{*}$} \\
\hline Ausência & $206(86,9)$ & $31(13,1)$ \\
\hline Presença & $165(80,9)$ & $39(19,1)$ \\
\hline \multicolumn{3}{|l|}{ Modelo demanda controle $(423)^{*}$} \\
\hline Baixa exigência & $126(92,6)$ & $10(7,4)$ \\
\hline Trabalho ativo & $78(77,2)$ & $23(22,8)$ \\
\hline Trabalho passivo & $82(82,0)$ & $18(18,0)$ \\
\hline Alta exigência & $68(79,1)$ & $18(20,9)$ \\
\hline \multicolumn{3}{|c|}{ Esforço envolvido na realização do trabalho (444)* } \\
\hline Baixo esforço & $202(90,1)$ & $22(9,9)$ \\
\hline Alto esforço & $173(78,6)$ & $47(21,4)$ \\
\hline \multicolumn{3}{|l|}{ Recompensas propiciadas pelo trabalho (438) } \\
\hline Alta recompensa & $205(83,3)$ & $41(16,7)$ \\
\hline Baixa recompensa & $163(84,8)$ & $29(15,2)$ \\
\hline
\end{tabular}

As maiores frequências de autoavaliação negativa do estado de saúde foram observadas entre as trabalhadoras que estavam satisfeitas com sua capacidade para o trabalho $(16,2 \%)$, entre as insatisfeitas com o trabalho $(19,1 \%)$, satisfeitas com o salário/renda (16\%), insatisfeitas com as relações pessoais $(18,6 \%)$, insatisfeitas consigo mesmas $(25 \%)$ e insatisfeitas com sua qualidade de vida $(25,4 \%)$ (Tabela 4). Foram selecionadas para a análise multivariada as variáveis: satisfação com o trabalho, satisfação consigo mesmo e avaliação da qualidade de vida. 
Autoavaliação da saúde segundo níveis de satisfação do trabalho e situação de saúde de trabalhadoras de enfermagem da atenção básica. Bahia, Brasil, 2011-2012

\begin{tabular}{|c|c|c|}
\hline \multirow[b]{2}{*}{ Variáveis (n) } & \multicolumn{2}{|c|}{ Autoavaliação do estado de saúde } \\
\hline & $\begin{array}{l}\text { Positiva } \\
\text { n (\%) }\end{array}$ & $\begin{array}{l}\text { Negativa } \\
\text { n (\%) }\end{array}$ \\
\hline \multicolumn{3}{|l|}{ Satisfação do trabalho } \\
\hline \multicolumn{3}{|c|}{ Satisfação com a capacidade para o trabalho (450) } \\
\hline Satisfeita & $296(83,8)$ & $57(16,2)$ \\
\hline Insatisfeita & $83(85,6)$ & $14(14,4)$ \\
\hline \multicolumn{3}{|l|}{ Satisfação com o trabalho (449)* } \\
\hline Satisfeita & $290(85,5)$ & $49(14,5)$ \\
\hline Insatisfeita & $89(80,9)$ & $21(19,1)$ \\
\hline \multicolumn{3}{|l|}{ Satisfação com salário/renda (447) } \\
\hline Satisfeita & $63(84)$ & $12(16)$ \\
\hline Insatisfeita & $314(84,4)$ & $58(15,6)$ \\
\hline \multicolumn{3}{|l|}{ Satisfação com as relações pessoais (450) } \\
\hline Satisfeita & $331(84,6)$ & $60(15,4)$ \\
\hline Insatisfeita & $48(81,4)$ & $11(18,6)$ \\
\hline \multicolumn{3}{|l|}{ Satisfação consigo mesma (451)* } \\
\hline $\begin{array}{l}\text { Satisfeita } \\
\text { Insatisfeita }\end{array}$ & $\begin{array}{l}329(85,9) \\
51(75)\end{array}$ & $\begin{array}{l}54(14,1) \\
17(25)\end{array}$ \\
\hline \multicolumn{3}{|l|}{ Avaliação da qualidade de vida (450)* } \\
\hline Satisfeita & $282(88,1)$ & $38(11,9)$ \\
\hline Insatisfeita & $97(74,6)$ & $33(25,4)$ \\
\hline \multicolumn{3}{|c|}{ Situação de saúde } \\
\hline \multicolumn{3}{|c|}{ Doenças ocupacionais ou profissionais $(444) *$} \\
\hline Não & $350(85,6)$ & $59(14,4)$ \\
\hline Sim & $23(65,7)$ & $12(34,3)$ \\
\hline \multicolumn{3}{|l|}{ Acidentes de trabalho $(445)^{*}$} \\
\hline Não & $359(85,3)$ & $62(14,7)$ \\
\hline Sim & $15(62,5)$ & $9(37,5)$ \\
\hline \multicolumn{3}{|l|}{ Licença médica no último ano (449) } \\
\hline N̦ão & $249(84,1)$ & $47(15,9)$ \\
\hline Sim & $129(84,3)$ & $24(15,7)$ \\
\hline \multicolumn{3}{|l|}{ Presença de comorbidade $(441)^{*}$} \\
\hline Não & $102(93,6)$ & $7(6,4)$ \\
\hline Sim & $269(81)$ & $63(19)$ \\
\hline \multicolumn{3}{|l|}{ Presença de dores osteomusculares (345)* } \\
\hline Não & $108(90)$ & $12(10)$ \\
\hline Sim & $185(82,2)$ & $40(17,8)$ \\
\hline \multicolumn{3}{|l|}{ Transtornos mentais comuns $(438)^{*}$} \\
\hline Não & $322(87,7)$ & $45(12,3)$ \\
\hline Sim & $46(64,8)$ & $25(35,2)$ \\
\hline
\end{tabular}

Ao abordar a situação de saúde, as maiores frequências de autoavaliação negativa foram encontradas entre as que referiram alguma doença ocupacional ou profissional $(34,3 \%)$, sofreram acidente de trabalho nos últimos 12 meses $(37,5 \%)$, apresentavam alguma comorbidade (19\%), dores osteomusculares $(17,8 \%)$ e TMC $(35,2 \%)$ (Tabela 4). As variáveis selecionadas para a análise multivariada foram: doenças ocupacionais ou profissionais, acidentes de trabalho, comorbidades, dores osteomusculares e TMC.

\section{Análise multivariada}

No modelo final, mantiveram-se estatisticamente associados à autoavaliação negativa do estado de saúde: incompatibilidade das atividades desenvolvidas (RP: 3,42; IC95\%: 1,79-6,52), média (RP: 3,00; IC95\%: 1,47-6,14) e alta sobrecarga doméstica (RP: 3,46; IC95\%: 1,73-6,91), situação de trabalho 
ativo segundo o Modelo Demanda-Controle (RP: 2,40; IC95\%: 1,22-4,73), insatisfação com a qualidade de vida (RP: 1,83; IC95\%: 1,18-2,82) e TMC (RP: 2,28; IC95\%: 1,45-3,57) (Tabela 5).

Tabela 5

Fatores associados à autoavaliação negativa da saúde em trabalhadoras de enfermagem da atenção básica na Bahia, Brasil, 2011 e 2012, obtidos em análise multivariada

\begin{tabular}{|c|c|c|c|}
\hline $\begin{array}{l}\text { Fatores associados à autoavaliação negativa do } \\
\text { estado de saúde }\end{array}$ & $\begin{array}{c}\text { RP } \\
\text { bruta }\end{array}$ & RP ajustada & IC95\% \\
\hline \multicolumn{4}{|l|}{ Compatibilidade das atividades desenvolvidas } \\
\hline Compatíveis com o cargo & 1,00 & 1,00 & \\
\hline Não compatíveis com o cargo & 2,46 & 3,42 & $1,79-6,52$ \\
\hline \multicolumn{4}{|l|}{ Sobrecarga doméstica } \\
\hline Baixa sobrecarga doméstica & 1,00 & 1,00 & \\
\hline Média sobrecarga doméstica & 2,36 & 3,00 & $1,47-6,14$ \\
\hline Alta sobrecarga doméstica & 2,93 & 3,46 & $1,73-6,91$ \\
\hline \multicolumn{4}{|l|}{ Modelo Demanda-Controle } \\
\hline Baixa exigência & 1,00 & 1,00 & \\
\hline Trabalho ativo & 3,08 & 2,40 & $1,22-4,73$ \\
\hline Trabalho passivo & 2,43 & 1,89 & $0,95-3,74$ \\
\hline Alta exigência & 2,82 & 1,94 & $0,94-4,02$ \\
\hline \multicolumn{4}{|l|}{ Avaliação da qualidade de vida } \\
\hline Satisfeita & 1,00 & 1,00 & \\
\hline Insatisfeita & 2,13 & 1,83 & $1,18-2,82$ \\
\hline \multicolumn{4}{|l|}{ Transtornos mentais comuns } \\
\hline Não & 1,00 & 1,00 & \\
\hline Sim & 2,86 & 2,28 & $1,45-3,57$ \\
\hline
\end{tabular}

Fonte: Os autores.

Nota: RP: razão de prevalência; IC95\%: intervalo de 95\% de confiança.

O modelo final apresentou um adequado poder de discriminação, ajustando-se bem aos dados (Teste de Hosmer e Lemeshow goodness of fit $=0,29$; Curva ROC $=0,79$ ), e não apresentou dados influentes.

\section{Prevalência de autoavaliação negativa do estado de saúde}

A prevalência estimada de autoavaliação negativa do estado de saúde entre trabalhadoras de enfermagem da Atenção Básica foi de 15,8\%, inferior às observadas entre trabalhadores de enfermagem de unidades hospitalares brasileiras: 19,5\% (Griep et al., 2011), 22,4\% (Theme-Filha, Costa e Guilam, 2013), 37,1\% (Porto et al., 2013) e internacionais: 58\% (Pappas, Alamanos e Dimoliatis, 2005) e 60,4\% (Malinauskiene et al., 2011).

É possível que as características do trabalho da enfermagem na Atenção Básica produzam danos reduzidos à saúde quando comparados à alta complexidade, devido às condições mais insalubres da segunda, tais como o contato constante com a morte e o sofrimento do paciente, que podem levar ao maior estresse ocupacional e ao risco de adoecimento. Esta hipótese pode ser reforçada pela prevalência reduzida de autoavaliação negativa do estado de saúde 
(5,6\%) encontrada em estudo na Macrorregião de Saúde do Triângulo Sul do Brasil com enfermeiros da Estratégia Saúde da Família (Fernandes et al., 2010).

Por outro lado, estudo com trabalhadores de centros de Atenção Primária à Saúde de Florianópolis-SC (Garcia, Höfelmann e Facchini, 2010) refuta essa ideia, na medida em que observam prevalências de autoavaliação negativa do estado de saúde de $21,69 \%$ entre enfermeiros e de $22,54 \%$ entre técnicos e auxiliares de enfermagem, maiores do que a encontrada neste estudo. Isto demonstra a necessidade de estudos longitudinais e comparativos que possibilitem avaliar a real associação entre autoavaliação negativa da saúde e as características do processo de trabalho nos diferentes serviços da Rede de Atenção a Saúde.

Essas contradições revelam a necessidade de uma interpretação cautelosa do indicador de autoavaliação da saúde, uma vez que pode ser influenciada pelos vieses de prevalência, de memória ou por expectativas de respostas socialmente aceitas, que se agravam quando se fala de trabalhadores, em que a doença é sugestiva de incapacidade e pode ocasionar o afastamento do trabalhador e, para os não estatutários, o desemprego.

Além disso, por se tratar de uma medida subjetiva, muitos aspectos pessoais e sociais estão associados à sua percepção. Neste estudo, alguns fatores foram identificados como associados à autoavaliação negativa do estado de saúde entre as trabalhadoras de enfermagem, a saber: incompatibilidade das atividades desenvolvidas, média e alta sobrecarga doméstica, situação de trabalho ativo segundo o Modelo Demanda-Controle, insatisfação com a qualidade de vida e TMC.

\section{Fatores associados à autoavaliação negativa do estado de saúde}

A incompatibilidade nas atividades desenvolvidas pela equipe de enfermagem da Atenção Básica pode gerar insatisfação, na medida em que esta tem consciência das atividades que devem e podem ser desempenhadas. Como exemplos, os atendimentos de urgência/emergência, nas unidades básicas de saúde baianas, onde não se encontra apoio suficiente e satisfatório para executar adequadamente o trabalho. Os profissionais deparam-se com circunstâncias que geram situações desfavoráveis para a equipe e para o usuário, refletindo em distanciamento entre o trabalho prescrito e o real. O que resulta em tensão, descontentamento e sofrimento do profissional (Almeida, 2012), com reflexo na sua saúde.

Os achados evidenciam, ainda, que as mulheres que desenvolvem trabalho doméstico estão mais expostas ao adoecimento, relação também encontrada em outros (Gomes e Mendes, 1995; Araújo, Pinho e Almeida, 2005; Pinho e 
Araújo, 2012). Nesta perspectiva, Fonseca et al. (2008) identificaram a influência da dupla jornada de trabalho (profissional e doméstico) na avaliação negativa da saúde, o que foi reafirmado neste estudo, ao identificar a sobrecarga doméstica como fator de exposição para a autoavaliação negativa do estado de saúde. Existem evidências consistentes na literatura (Araújo, Pinho e Almeida, 2005; Pinho e Araújo, 2012; Fernandes et al., 2013) da influência do trabalho doméstico e/ou da sobrecarga doméstica na saúde física e mental de mulheres, que podem ser explicadas pela sobrecarga de trabalho somada a desvalorização e não remuneração dessa atividade.

Além disso, a influência dos aspectos psicossociais do trabalho na autoavaliação de saúde é demonstrada em estudos da área (Costa, 2010; Mccarthy, Power e Greiner, 2010; Malinauskiene et al., 2011; Griep et al., 2011; Silva e Barreto, 2012; Theme-Filha, Costa e Guilam, 2013; Rocha et al., 2014), o que também foi observado nesta pesquisa. Em estudos de Costa (2010) e Malinauskiene et al. (2011), a situação de trabalho com maiores níveis de autoavaliação negativa da saúde foi a de alta exigência, o que coaduna com a predição do Modelo Demanda-Controle de Karasek. Entretanto, no presente estudo foi o trabalho ativo que apresentou maiores prevalências de autoavaliação negativa da saúde, o que evidencia que o alto controle não foi capaz de minimizar as cargas geradas pela alta demanda.

De forma similar, em estudo com profissionais de enfermagem (Griep et al., 2011) observou-se um menor poder preditivo da dimensão controle em ocupações que apresentam níveis muito elevados de demanda psicológica. A demanda psicológica refere-se ao desgaste psicológico decorrente das exigências necessárias para realização das atividades laborais, inclui pressão do tempo, nível de concentração requerida, interrupção das tarefas e dependência de atividades realizadas por outros trabalhadores (Karasek, 1979).

Este estudo revela que a sobrecarga de trabalho é prejudicial, mesmo quando há autonomia relativa da enfermagem na execução do seu processo de trabalho. Ou seja, em casos em que a demanda psicológica é muito alta, o controle sobre o próprio trabalho perde seu efeito moderador sobre os impactos negativos decorrentes desta. O que reafirma a importância fundamental dos aspectos psicossociais do trabalho no adoecimento do indivíduo, já tratado por Dejours (1994) no âmbito da Psicodinâmica do Trabalho.

As equipes de saúde dos serviços de atenção básica são constantemente cobradas pelas Secretarias Municipais de Saúde para o cumprimento de metas estabelecidas, embora não forneçam apoio financeiro, técnico ou educativo ao desenvolvimento desse processo (Almeida, 2012). Isso gera um aumento da demanda de trabalho e um controle que não existe, já que, muitas vezes, não se tem governabilidade e, em outras tantas, não há equipamentos para o desenvolvimento do que foi planejado, quer seja pela indisponibilidade 
de material ou pela falta de manutenção dos mesmos, o que acarreta esforço elevado para a realização deste trabalho.

Outro fator que pode ter influenciado na não observação de associação entre autoavaliação negativa de saúde e o trabalho de alta exigência refere-se ao pequeno número de trabalhadoras neste grupo $(\mathrm{n}=86)$ - o que pode ter afetado o nível de significância estatística -, uma vez que a razão de prevalência ajustada (modelo final de regressão logística) foi igual a 1,94, o que indica uma prevalência de autoavaliação negativa da saúde $94 \%$ maior entre as trabalhadoras expostas ao trabalho de alta exigência, quando comparados às de baixa exigência.

Diferenças significativas em relação à autoavaliação de saúde e indicadores de satisfação e morbidades também foram observadas neste estudo. A insatisfação com a qualidade de vida esteve associada com a autoavaliação negativa, que corrobora com outros estudos (Costa, 2010; Fernandes et al., 2010; Porto et al., 2013; Theme-Filha, Costa e Guilam, 2013).

A qualidade de vida é um construto de extrema complexidade e caracteriza, de alguma maneira, a forma como o indivíduo percebe sua vida, estando incluso nesta vertente a saúde, uma vez que essa é a primeira e essencial condição para que alguém possa considerar sua vida como tendo qualidade. Pode-se acrescentar que a autoavaliação desta condição resulta das potencialidades ou limitações impostas pela qualidade de vida do indivíduo e por suas condições biopsíquica (Porto et al., 2013).

A suspeição de TMC esteve associada a autoavaliação negativa da saúde entre as trabalhadoras de enfermagem da Atenção Básica (RP: 2,28; IC95\%: 1,45-3,57), mesmo após ajuste pelas outras variáveis associadas ao desfecho. Não foi encontrado na literatura disponível estudo específico que confirme essa associação, apesar de Dejours (1994) já ter afirmado esta relação em seus estudos sobre doenças psicossomáticas no trabalho. Outros estudos corroboram, ao identificar associações com desordens mentais em geral (Malinauskiene et al., 2011) e com sintomas específicos, por exemplo, depressivos e ansiosos (Lima-Costa, Firmo e Uchôa, 2004), tensão psicológica (Höfelmann e Blank, 2007) e níveis de estresse (Fonseca et al., 2008; Silva, 2012).

Embora sejam eventos de baixa letalidade, os TMCs causam prejuízos nas atividades diárias do indivíduo e são responsáveis por sofrimento, incapacidade e absenteísmo no trabalho (Carvalho et al., 2013). Assim, o sofrimento mental é capaz de ocasionar danos às funções físicas dos indivíduos, através da ocorrência de doenças psicossomáticas (Capitão e Carvalho, 2006). Reafirmando que a saúde e a autopercepção sobre ela depende também das questões psíquicas.

As morbidades físicas ou mentais são condições individuais que podem acarretar incapacidades, e estão estritamente relacionadas à percepção de saúde. 
Desta forma, a autoavaliação reflete uma percepção subjetiva e integrada de saúde do indivíduo, incluindo suas dimensões biológicas, psicológicas e sociais, que não são perceptíveis por observadores externos.

\section{Conclusão}

Neste estudo, evidenciaram-se, entre os fatores associados à autoavaliação do estado de saúde em trabalhadoras de enfermagem da Atenção Básica, a incompatibilidade das atividades desenvolvidas com o cargo, a sobrecarga doméstica (média e alta), a situação de trabalho ativo segundo o modelo demanda-controle, insatisfação com a qualidade de vida e a suspeição de TMC.

Este estudo, devido ao seu desenho transversal, apresenta como limite a impossibilidade de estabelecimento de relações causais, sendo possível apenas identificar associações entre os fatores estudados, visto que os dados sobre exposição e desfecho foram coletados simultaneamente. Por se tratar de um estudo ocupacional, pode estar sujeito ao efeito do trabalhador sadio, uma vez que há a possibilidade de indivíduos com precárias condições de saúde terem sido demitidos ou afastados do trabalho, o que pode ter reduzido a prevalência de autoavaliação negativa de saúde encontrada. Outra questão que merece reflexão é o fato de a doença sugerir incapacidade e pode ocasionar o afastamento do trabalho ou desemprego, fazendo com que autoavaliar a saúde negativamente não seja socialmente encorajado ou aprovado.

Os resultados aqui encontrados apontam que as características pessoais foram menos expressivas na explicação do fenômeno, observando-se relação mais consistente com as características relacionadas ao trabalho. O que alerta para a importância de ações específicas de saúde do trabalhador na esfera da prevenção e promoção da saúde, com ênfase nos fatores de exposição identificados.

Os resultados apresentados podem subsidiar outras reflexões acerca da saúde dos trabalhadores do setor e da qualidade da assistência prestada por eles. Levantando-se a hipótese, a ser testada, de que se o trabalho da enfermagem for prestado por profissionais insatisfeitos e/ou doentes terá impactos também negativos na qualidade do serviço ofertado. 


\section{Colaboradores}

Iracema Lua foi responsável pela redação do manuscrito, concepção, desenho, análise e interpretação dos dados da pesquisa; Maura Maria Guimarães de Almeida colaborou na concepção do estudo, elaboração e revisão crítica do manuscrito e aprovação da final; Tânia Maria de Araújo foi coordenadora do projeto de pesquisa original, participou na elaboração e revisão crítica do manuscrito, e aprovação da versão final; Jorgana Fernanda de Souza Soares colaborou na elaboração e revisão crítica do manuscrito e aprovação da versão final; Kionna Oliveira Bernardes Santos colaborou no planejamento do estudo, análise e interpretação dos dados da pesquisa; aprovação da versão final.

\section{Agradecimentos}

Ao Conselho Nacional de Desenvolvimento Científico e Tecnológico (CNPq - Edital Universal - Processo 480611/2010-62010), Fundação de Amparo à Pesquisa do Estado da Bahia (Fapesb) pelo financiamento da pesquisa (TO RED0025/2013 Redes de Pesquisa e PPSUS TO 022/2014) e Coordenação de Aperfeiçoamento de Pessoal de Nível Superior (Capes) pela concessão de bolsa de mestrado.

Resumen El objetivo de este estudio es evaluar los factores asociados a la autoevaluación negativa de la salud entre trabajadoras de enfermería. Se realizó un estudio transversal exploratorio, a partir de una muestra probabilística de 451 trabajadoras de enfermería de la Atención Básica de la Salud. Se aplicó un cuestionario con información sociodemográfica, de hábitos de vida, laborales, aspectos psicosociales y satisfacción en el trabajo, y morbilidad de las trabajadoras. La autoevaluación de la salud fue realizada a través de la pregunta “¿De manera general, en comparación a las personas de su edad, cómo considera usted su propio estado de salud?". Los datos se analizaron por medio del modelo de regresión logística en bloques. Se identificó que la autoevaluación negativa de la salud prevalece con un 15,8\%. Los factores asociados fueron: incompatibilidad de las actividades desarrolladas en relación al cargo, media y alta sobrecarga doméstica, situación de trabajo activo (alto control, alta demanda), mala evaluación de la calidad de vida y trastornos mentales comunes. Los resultados corroboran la relación entre el proceso salud-enfermedad y las condiciones de vida y trabajo, e indican la necesidad de políticas públicas de prevención y promoción de la salud de las trabajadoras de enfermería. Estas políticas, al constituir situaciones favorables de trabajo, pueden ser muy importantes para la calidad de la asistencia brindada a los usuarios del Sistema Único de Salud.

Palabras clave autoevaluación; enfermería; atención básica de la salud; salud del trabajador; trabajo femenino. 


\section{Notas}

${ }^{1}$ Universidade Estadual de Feira de Santana, Feira de Santana, Bahia. Brasil. <ira_lua@hotmail.com>

Correspondência: Universidade Estadual de Feira de Santana, Departamento de Saúde, Novo Horizonte, CEP 44036-900, Feira de Santana, Bahia, Brasil.

${ }^{2}$ Universidade Estadual de Feira de Santana, Programa de Pós-Graduação em Saúde Coletiva, Feira de Santana, Bahia, Brasil.

$<$ mesauco@hotmail.com>

${ }^{3}$ Universidade Estadual de Feira de Santana, Programa de Pós-Graduação em Saúde Coletiva, Feira de Santana, Bahia, Brasil.

<araujo.tania@uefs.br>

${ }^{4}$ Universidade Federal da Bahia, Faculdade de Medicina da Bahia, Salvador, Bahia, Brasil. <jfss_rs@hotmail.com>

${ }^{5}$ Universidade Federal da Bahia, Instituto de Ciências da Saúde, Salvador, Bahia, Brasil. $<$ kionnabernardes@hotmail.com>

\section{Referências}

ALMEIDA, Tatiane S. C. Repercussões do processo de trabalho na saúde do enfermeiro do Programa Saúde da Família: prazer e sofrimento. 168 f. Dissertação (Mestrado) - Universidade Estadual de Feira de Santana, Bahia, 2012.

AQUINO, Estela M. L. Gênero, trabalho e hipertensão arterial: um estudo de trabalhadoras de enfermagem em Salvador. 1996. 159f. Tese (Doutorado em Saúde Coletiva) - Universidade Federal da Bahia, Bahia, 1996. 
ARAÚJO, Tânia M. et al. Aspectos psicossociais do trabalho e distúrbios psíquicos entre trabalhadoras de enfermagem. Revista de Saúde Pública, São Paulo, v. 37, n. 4, p. 424-433, 2003. ARAÚJO, Tânia M.; PINHO, Paloma D. S.; ALMEIDA, Maura M. G. Prevalência de transtornos mentais comuns em mulheres e sua relação com as características sociodemográficas e o trabalho doméstico. Revista Brasileira de Saúde Materno Infantil, Recife, v. 5, n. 3, p. 337-348, 2005.

BRASIL. Ministério da Saúde. Política Nacional de Atenção Básica. Brasília: Ministério da Saúde, 2012.

BRYŁA, Marek; SZYMOCHA, Monika; MANIECKA-BRYŁA, Irena. Determinants of self-rated health of a working population. Medycyna Pracy, Lódiz, v. 66, n. 1, p. 17-28, 2015.

CAPITÃO, Claúdio G.; CARVALHO, Érica B. Psicossomática: duas abordagens de um mesmo problema. Revista de Psicologia da Vetor Editora, São Paulo, v. 7, n. 2, p. 21-29, 2006.

CARVALHO, Carla N. et al. Prevalência e fatores associados aos transtornos mentais comuns em residentes médicos e da área multiprofissional. Jornal Brasileiro de Psiquiatria, Rio de Janeiro, v. 62, n. 1, p. 38-45, 2013.

COSTA, Maria A. S. O estresse no trabalho e autoavaliação da saúde entre os trabalhadores da enfermagem das unidades de urgências e emergências da Secretaria Municipal de Saúde de Campo Grande/MS, 2010. 2010. 66 f. Dissertação (Mestrado em Saúde Pública) - Escola Nacional de Saúde Pública Sergio Arouca, Rio de Janeiro, 2010.

COUTINHO, Leticia M. S.; SCAZUFCA, Marcia; MENEZES, Paulo R. Métodos para estimar razão de prevalência em estudos de corte transversa. Revista de Saúde Pública, São Paulo, v. 42, n. 6, p. 992-998, 2008.

DEJOURS, Christophe. Psicodinâmica do trabalho: contribuição da Escola Dejuriana à analise da relação prazer, sofrimento e trabalho. São Paulo: Atlas, 1994.

ERGIN, Isil; MANDIRACIOGLU, Aliye. Demographic and socioeconomic inequalities for self-rated health and happiness in elderly: The situation for Turkey regarding World Values Survey between 1990 and 2013. Archives of Gerontology and Geriatrics, Ireland, v. 61, p. 224-230, 2015.

FERNANDES, Janielle S. et al. Qualidade de vida dos enfermeiros das equipes de saúde da família: a relação das variáveis sociodemográficas. Texto Contexto Enfermagem, Florianópolis, v. 19, n. 3, p. 434-442, 2010.

FERNANDES, Juliana D. C. et al. Jornada de trabalho e comportamentos de saúde entre enfermeiros de hospitais públicos. Revista Latino-Americana de Enfermagem, São Paulo, v. 21, n. 5, p. $8,2013$.

FONSECA, Silvio A. et al. Percepção de saúde e fatores associados em industriários de Santa Catarina, Brasil. Cadernos de Saúde Pública, Rio de Janeiro, v. 24, n. 3, p. 567-576, 2008.

FRANCISCO, Priscila M. S. B. et al. Medidas de associação em estudo transversal com delineamento complexo: razão de chances e razão de prevalência. Revista Brasileira de Epidemiologia, São Paulo, v. 11, n. 3, p. $347-$ 355, 2008.

GARCIA, Leila P.; HÖFELMANN, Doroteia A.; FACCHINI, Luiz A. Self-rated health and working conditions among workers from primary health care centers in Brazil. Cadernos de Saúde Pública, Rio de Janeiro, v. 26, n. 5, p. 971-980, 2010.

GOMES, Daisy L. S.; MENDES, Iranilde J. M. A força de trabalho da mulher. Acta Paulista de Enfermagem, São Paulo, v. 8, n. 1, p. 61-74, 1995.

GRIEP, Rosane H. et al. Uso combinado de modelos de estresse no trabalho e a saúde autoerreferida na enfermagem. Revista de Saúde Pública, São Paulo, v. 45, n. 1, p. 145152, 2011. 
GUIMARÃES, Joanna M. N. et al. Association between self-rated health and mortality: 10 years follow-up to the Pró-Saúde cohort study. BMC Public Health, London, v. 12, p. 1-10, 2012.

HÄMMIG, Oliver; BAUER, Georg F. Work, work-life conflict and health in an industrial work environment. Occupational Medicine, London, v. 64, p. 34-38, 2014.

HÖFELMANN, Doroteia A.; BLANK, Nelson. Autoavaliação de saúde entre trabalhadores de uma indústria no sul do Brasil. Revista de Saúde Pública, São Paulo, v. 41, n. 5, p. 777-787, 2007.

KARASEK, Robert A. Job Demand, job decision latitude, and mental strain: implications for job redesign. Administrative Science Quarterly, Nova Iorque, v. 24, p. 285-308, 1979.

LIMA-COSTA, Maria F.; FIRMO, Josélia O. A.; UCHÔA, Elisabeth. A estrutura da autoavaliação da saúde entre idosos: Projeto Bambuí. Revista de Saúde Pública, São Paulo, v. 38, n. 6, p. 827-834, 2004.

MALINAUSKIENE, Vilija et al. Associations between self-rated health and psychosocial conditions, lifestyle factors and health resources among hospital nurses in Lithuania. Journal of Advanced Nursing, v. 67, n. 11, p. 23832393, 2011.

MCCARTHY, Vera J. C.; POWER, S.; GREINER, Birgit A. Perceived occupational stress in nurses working in Ireland. Occupational Medicine, Oxford, v. 60, p. 604-610, dez. 2010.

PAGOTTO,Valeria; NAKATANI, Adélia Y. K.; SILVEIRA, Érika A. Fatores associados à autoavaliação de saúde ruim em idosos usuários do Sistema Único de Saúde. Cadernos de Saúde Pública, Rio de Janeiro, v. 27, n. 8, p. 1593-1602, 2011.

PAPPAS, Noula A.; ALAMANOS, Yannis; DIMOLIATIS, Loannis D. K. Self-rated health, work characteristics and health related behaviours among nurses in Greece: a cross sectional study.
BioMed Central Nursing, Bethesda-Maryland, v. 4, p. 1-8, 2005.

PAVÃO, Ana L. B.; WERNECK, Guilherme L.; CAMPOS, Mônica R. Autoavaliação do estado de saúde e a associação com fatores sociodemográficos, hábitos de vida e morbidade na população: um inquérito nacional. Cadernos de Saúde Pública, Rio de Janeiro, v. 29, n. 4, p. 723-734, 2013.

PERES, Marco A. et al. Autoavaliação da saúde em adultos no Sul do Brasil. Revista de Saúde Pública, São Paulo, v. 44, n. 5, p. 901-911, 2010.

PETARLI, Glenda B. et al. Autoavaliação do estado de saúde e fatores associados: um estudo em trabalhadores bancários. Cadernos de Saúde Pública, Rio de Janeiro, v. 31, n. 4, p. 787-799, 2015.

PINHO, Paloma D. S.; ARAÚJO, Tânia M. Associação entre sobrecarga doméstica e transtornos mentais comuns em mulheres. Revista Brasileira de Epidemiologia, São Paulo, v. 15, n. 3, p. 560-572, 2012.

PORTO, Adrize R. et al. Autoavaliação de saúde e doenças crônicas entre enfermeiros de Pelotas/RS. Revista Eletrônica de Enfermagem, Goiânia, v. 15, n. 3, p. 763-771, 2013.

ROCHA, Kátia B. et al. Clase social, factores de riesgo psicosocial en el trabajo y su asociación con la salud autopercibida y mental en Chile. Cadernos de Saúde Pública, Rio de Janeiro, v. 30, n. 10, p. 2.219-2.234, 2014.

SANTOS, K. O. B. et al. Avaliação de um instrumento de mensuração de morbidade psíquica: Estudo de validação do Self-Reporting Questionnaire (SRQ-20). Revista Baiana de Saúde Pública, Salvador, v. 34, n. 3, p. 544560, 2010.

SILVA, Diego A. S. Indicadores do estilo de vida e autoavaliação negativa de saúde em universitários de uma instituição pública do nordeste do Brasil. Revista Brasileira de Atividade Física e Saúde, Florianópolis, v. 17, n. 4, p. 263-269, 2012. 
SILVA, Luiz S.; BARRETO, Sandhi M. Stressful working conditions and poor self-rated health among financial services employees. Revista de Saúde Pública, São Paulo, v. 46, n. 3, p. 407-416, 2012.

THEME FILHA, Mariza M.; COSTA, Maria A. S.; GUILAM, Maria C. R. Estresse ocupacional e autoavaliação de saúde entre profissionais de enfermagem. Revista Latino-Americana de Enfermagem, São Paulo, v. 21, n. 2, 2013.

TRAEBERT, Jefferson; BORTOLUZZI, Marcelo C.; KEHRIG, Ruth T. Autopercepção das con- dições de saúde da população adulta, sul do Brasil. Revista de Saúde Pública, São Paulo, v. 45, n. 4, p. 789-793, 2011.

WU, Shunquan et al. The relationship between self-rated health and objective health status: a population-based study. BMC Public Health, London, v. 13, p. 1-10, 2013.

Recebido em 29/03/2016.

Aprovado em 27/08/2017. 